\title{
Enantioselective synthesis of a 2,3-benzodiazepine intermediate of BET inhibitor BAY 1238097 via catalytic asymmetric hydrogenation Supporting Information
}

Gerard K.M. Verzijla, Jorma Hassfeld ${ }^{\mathrm{b}}$, André H.M. de Vries ${ }^{\mathrm{a}}$ and Laurent Lefort ${ }^{\mathrm{a}}$ *

a InnoSyn B.V., Urmonderbaan 22, 6167 RD Geleen, The Netherlands

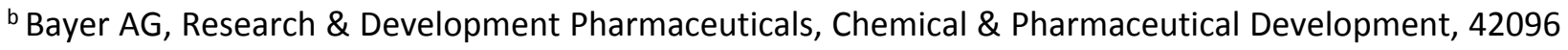
Wuppertal, Germany

\section{Contents}

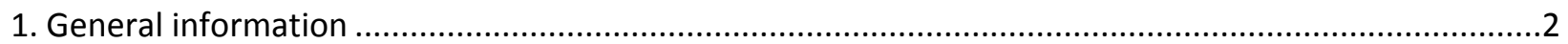

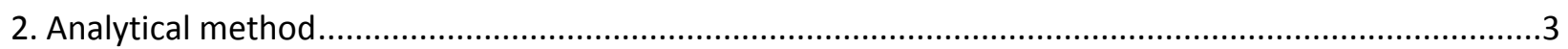

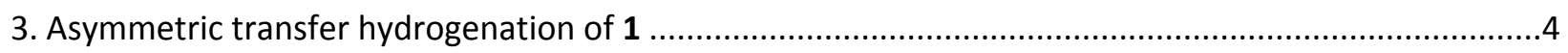

4. First screening for the enantioselective hydrogenation of 1 .........................................................

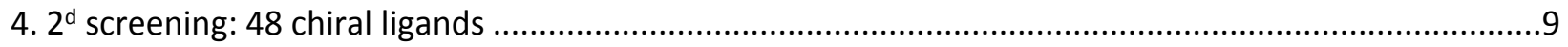

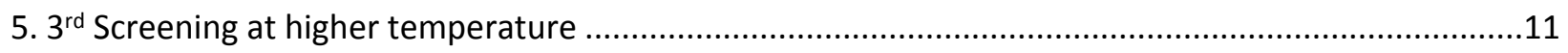

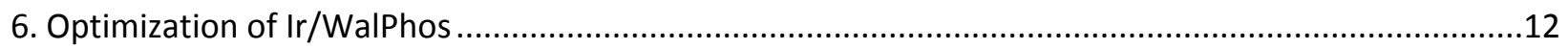

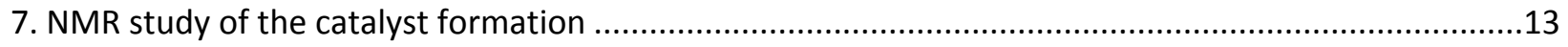

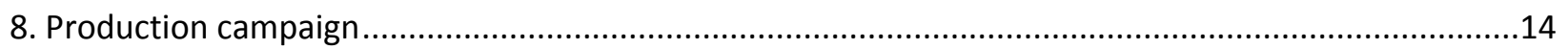




\section{General information}

All laboratory scale reactions were performed in a dry nitrogen atmosphere using standard Schlenk techniques or in the glove box. Solvents used in these experiments were reagent grade or better. Dry DCM and THF were obtained from MBraun SPS system. $[\operatorname{Ir}(\mathrm{COD}) \mathrm{Cl}]_{2}$ was purchased from Umicore and Heraeus. Ligands were purchased at Aldrich or Strem.

The libraries of catalysts were prepared using a Zinsser Lissy liquid handling robot equipped with 4 syringes and placed inside a glove box (See picture below).

The hydrogenation reaction is carried out in a Premex 96-Multi Reactor ${ }^{1}$ that can accommodate 96 reactions vessels at the same temperature and hydrogen pressure (See picture below).

${ }^{31} \mathrm{P}$ NMR spectra were recorded on a Bruker Advance $300(300 \mathrm{MHz})$ NMR spectrometer and reported in units of parts per million (ppm).
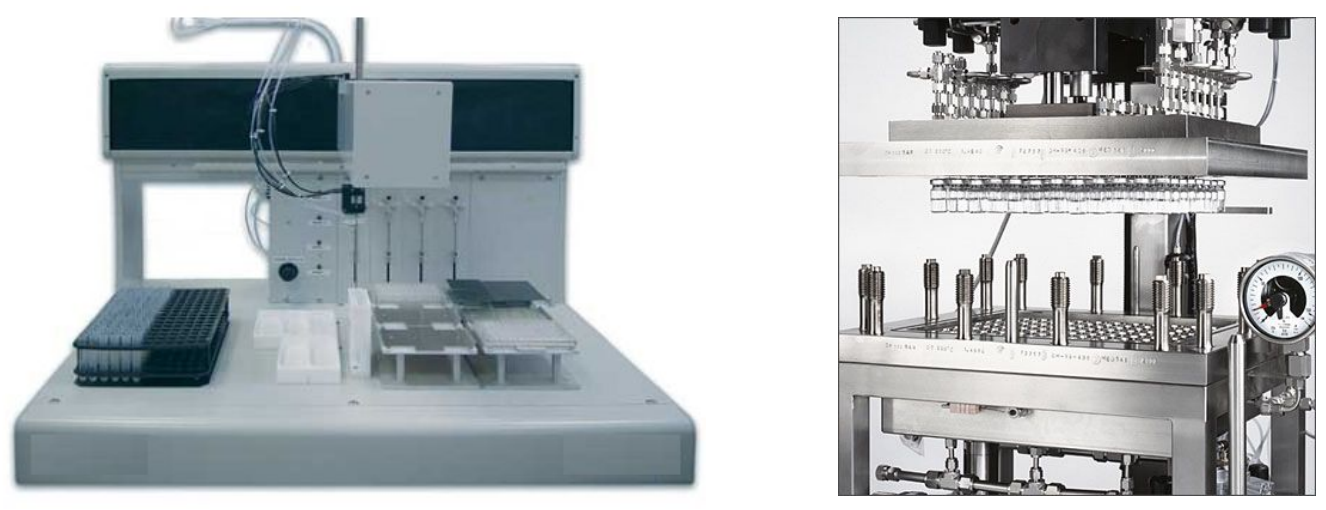

Figure S1. Hardware available at InnoSyn for high throughput screening: Liquid handling robot (Zinsser Lissy, left) and Premex 96-Multi Reactor (right). 


\section{Analytical method}

Conversion and enantiomeric excess were determined by HPLC (Agilent system equipped with a UV-Vis Detector).

Column: $\quad$ Chiralpak ID $250 \mathrm{~mm} \times 4.6 \mathrm{~mm}$ ID $5 \mu \mathrm{m}$

Mobile phase: $\quad 75 \mathrm{v} / \mathrm{v}$ n-Heptane; $0.1 \% \mathrm{v} / \mathrm{v}$ diethylamine

Flow: $\quad 1.7 \mathrm{~mL} / \mathrm{min}$

Oven: $\quad 35^{\circ} \mathrm{C}$

Injection volume : $\quad 4 \mu \mathrm{L}$

Detection : $\quad$ UV wavelength $256 \mathrm{~nm}$

Analysis time : $\quad 20 \mathrm{~min}$

Substrate and racemate were dissolved in $\mathrm{EtOH} / \mathrm{MeOH} 1 / 1$ (v/v)

Concentrations: Substrate 1: $2.306 \mathrm{mg} / \mathrm{mL}$; Racemate 2: $2.08 \mathrm{mg} / \mathrm{mL}$

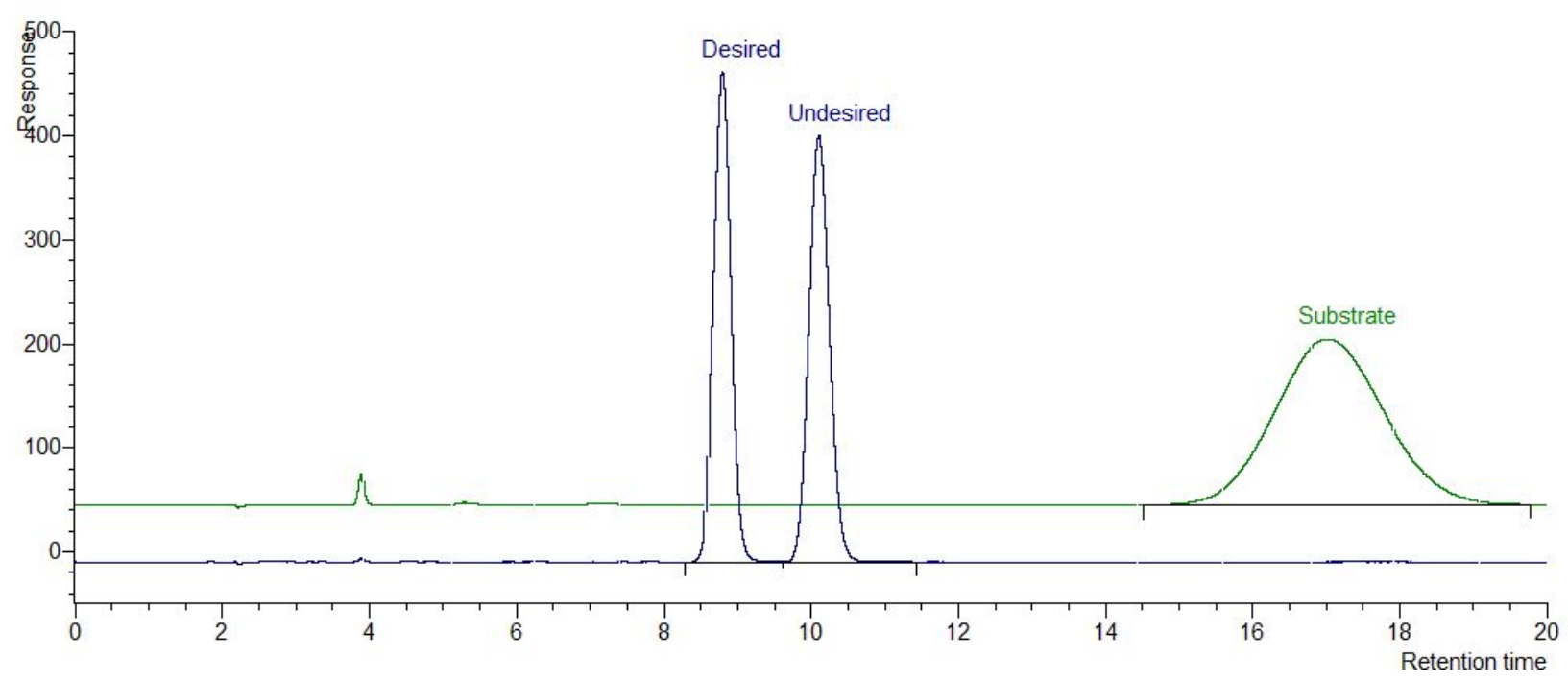

Figure S2. HPLC chromatograms for the reference material

$\begin{array}{lcc} & \text { RT }(\mathbf{m i n}) & \text { RRT } \\ \text { Desired enantiomer }(S) & 8.81 & 1.00 \\ \text { Undesired enantiomer }(R) & 10.12 & 1.15 \\ \text { Substrate } & 17.03 & 1.93\end{array}$




\section{Asymmetric transfer hydrogenation of 1}

List of catalysts tested in transfer hydrogenation:

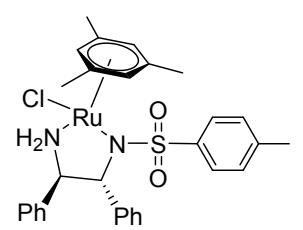

A: $\operatorname{RuCl}[(\mathrm{R}, \mathrm{R})-\mathrm{TsDPen}]$ (mesitylene)

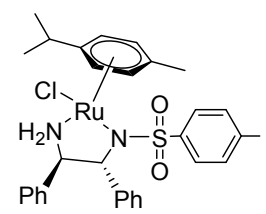

F: RuCI[(R,R)-TsDPen] (cymene)

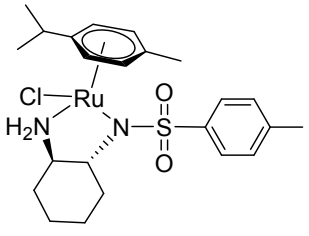

B: RuCl[(R,R)-TsDach] (cymene)

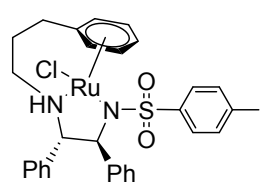

G: RuCl[(S,S)-teth-TsDPen]

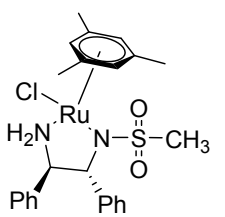

C: $\mathrm{RuCl}[(\mathrm{R}, \mathrm{R})-\mathrm{MsDPen}]$ (mesitylene)

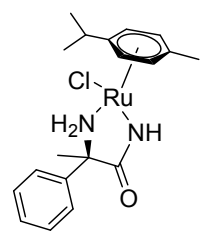

D: RuCl[(R)-2-Ph-2-amino propionamide](cymene)

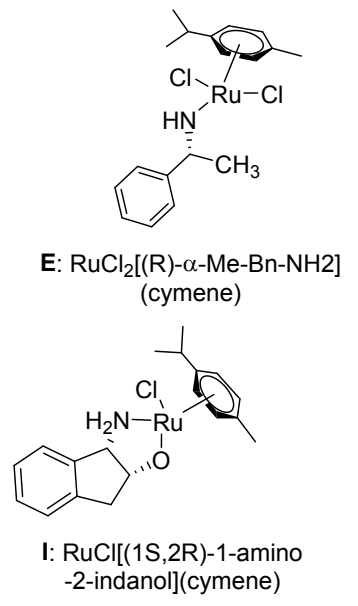

Catalysts Preparation: Catalysts A, B, C, F and $\mathbf{G}$ are commercially available and were used as such. The catalysts $\mathbf{D}$ and $\mathbf{E}$ were prepared according to the following procedure: $\left[\mathrm{RuCl}_{2} \text { cymene }\right]_{2}(61.24 \mathrm{mg}, 0.1$ $\mathrm{mmol}$ ) and the ligand (0.2 mmol, (R)-2-phenyl propionamide (33 mg, $0.2 \mathrm{mmol}$ ) for $\mathbf{D} ;(R)-\alpha-\mathrm{Me}-\mathrm{Bn}-\mathrm{NH}_{2}$ (24.2 $\mathrm{mg}, 0.2 \mathrm{mmol})$ for $\mathrm{E})$ are dissolved in $\mathrm{DCM}(4 \mathrm{~mL})$ containing $\mathrm{Et}_{3} \mathrm{~N}(100 \mu \mathrm{L})$ and heated to $50^{\circ} \mathrm{C}$ for 0.25 hour in a sealed vial. The mixture is cooled to room temperature and extracted with a $4 \mathrm{M} \mathrm{KOH}$ solution in water $(1 \mathrm{~mL})$. The DCM phase was recovered, dried over $\mathrm{Na}_{2} \mathrm{SO}_{4}$, filtrated. Upon removal of DCM, a dark red crystalline solid is obtained for $\mathbf{D}$, a brown viscous oil for $\mathbf{E}$. Both catalysts were used as such. The catalysts $\mathbf{H}$ and I were prepared according to the following procedure: $\left[\mathrm{RuCl}_{2} \mathrm{Cymene}_{2}(10 \mathrm{mg}\right.$, $0.0163 \mathrm{mmol})$ and the ligand $(0.1 \mathrm{mmol})$ were heated in IPA $(0.5 \mathrm{~mL})$ at $50^{\circ} \mathrm{C}$ for 0.25 hour. $100 \mu \mathrm{L}$ of the Ru solution $(0.00625 \mathrm{mmol})$ was used as such in the transfer hydrogenation experiments.

\section{Reaction Conditions:}

\section{Conditions 1: $\mathrm{KOH} / \mathrm{MeOH} / \mathrm{HCOOH}$}

A $1 \mathrm{M}$ solution of $\mathrm{KOH}$ in $\mathrm{MeOH}(1 \mathrm{~mL})$ was diluted with $\mathrm{MeOH}(1 \mathrm{~mL})$. The solution was treated by formic acid and added to a $5 \mathrm{~mL}$ vial charged with substrate $1(37.3 \mathrm{mg}, 0.1 \mathrm{mmol}$ ) followed by the addition of the catalyst $(0.00625 \mathrm{mmol})$. The vial was sealed, degassed by $\mathrm{N}_{2}$ and heated to $40^{\circ} \mathrm{C}$. The substrate was not completely dissolved. 


\section{Conditions 2: TEAF/MeOH}

A solution of TEAF 1:1 (Et $\left.{ }_{3} \mathrm{~N}: \mathrm{HCOOH}, 85 \mathrm{mg}, 0.57 \mathrm{mmol} \mathrm{HCOOH}\right)$ or TEAF 5:2 (49 mg, $\left.0.57 \mathrm{mmol} \mathrm{HCOOH}\right)$ in $\mathrm{MeOH}(2 \mathrm{~mL}$ ) was added to a $5 \mathrm{~mL}$ vial charged with benzodiazepine 1 ( $37.3 \mathrm{mg}, 0.1 \mathrm{mmol}$ ) followed by the addition of the catalyst $(0.00625 \mathrm{mmol})$. The vial was sealed, degassed by $\mathrm{N}_{2}$ and heated to $40^{\circ} \mathrm{C}$. The substrate was not completely dissolved.

\section{Conditions 3: $\mathrm{TEAF} / \mathrm{CH}_{2} \underline{\mathrm{Cl}}_{2}$}

In a $5 \mathrm{~mL}$ vial, benzodiazepine $1(37.3 \mathrm{mg}, 0.1 \mathrm{mmol})$ was dissolved in a mixture of TEAF 1:1 (85 $\mathrm{mg}, 0.57$ $\mathrm{mmol} \mathrm{HCOOH})$ and $\mathrm{CH}_{2} \mathrm{Cl}_{2}(2 \mathrm{~mL})$ followed by the addition of the catalyst $(0.00625 \mathrm{mmol})$. The mixture was degassed by $\mathrm{N}_{2}$ and heated to $40^{\circ} \mathrm{C}$. TEAF 1:1 does not properly dissolve (droplets on the vial wall).

\section{Conditions 4: $\mathrm{MeOH} / \mathrm{H}_{2} \mathrm{O}$ and $\mathrm{HCOONa}$}

A solution of HCOONa $(1.36 \mathrm{~g}, 20 \mathrm{mmol})$ in water $(15 \mathrm{~mL})$ was diluted with $\mathrm{MeOH}(25 \mathrm{~mL})$ giving a homogeneous solution. $2 \mathrm{~mL}$ of this solution ( $1 \mathrm{mmol} \mathrm{HCOONa}$ ) was added to a $5 \mathrm{~mL}$ vial charged with benzodiazepine 1 ( $37.3 \mathrm{mg}, 0.1 \mathrm{mmol}$ ) followed by the addition of the catalyst $(0.00625 \mathrm{mmol})$. The vial was sealed, degassed by $\mathrm{N}_{2}$ and heated to $40^{\circ} \mathrm{C}$. The substrate does not dissolve entirely.

\section{Conditions 5: Biphasic $\mathrm{HCOONa} / \mathrm{H}_{2} \mathrm{O}$ and $\mathrm{CH}_{2} \underline{\mathrm{Cl}}_{2}$}

HCOONa (1.36 g, $20 \mathrm{mmol}$ ) was dissolved in water $(10 \mathrm{~mL})$. In a $5 \mathrm{~mL}$ vial, benzodiazepine 1 (37.3 mg, 0.1 mmol) was dissolved in $\mathrm{CH}_{2} \mathrm{Cl}_{2}(1.5 \mathrm{~mL})$ followed by the addition of the aqueous solution of $\mathrm{HCOONa}(0.5$ $\mathrm{mL}, 1 \mathrm{mmol} \mathrm{HCOONa}$ ) and catalyst $(0.00625 \mathrm{mmol})$. The mixture was degassed by $\mathrm{N}_{2}$ and heated to $40^{\circ} \mathrm{C}$.

\section{Conditions 6: Biphasic withTEAF/ $\mathrm{H}_{2} \mathrm{O}$ and $\mathrm{CH}_{2} \underline{\mathrm{Cl}_{2}}$}

In a $5 \mathrm{~mL}$ vial, benzodiazepine $1(37.3 \mathrm{mg}, 0.1 \mathrm{mmol})$ was dissolved in $\mathrm{CH}_{2} \mathrm{Cl}_{2}(1.5 \mathrm{~mL})$ followed by the addition of TEAF 1:1 (85 mg, $0.57 \mathrm{mmol})$, water $(0.5 \mathrm{~mL})$ and catalyst $(0.00625 \mathrm{mmol})$. The mixture was degassed by $\mathrm{N}_{2}$ and heated to $40^{\circ} \mathrm{C}$.

\section{Conditions 7: IPA/KOtBu}

A solution of the Ru-catalyst $(0.00625 \mathrm{mmol})$ in IPA $(2 \mathrm{~mL})$ was prepared and treated with $\mathrm{KOtBu}(2.7 \mathrm{mg}$, $0.024 \mathrm{mmol})$. The solution was added to benzodiazepine 1 (37.3 $\mathrm{mg}, 0.1 \mathrm{mmol}, \mathrm{S} / \mathrm{C} 15)$ in a $5 \mathrm{~mL}$ vial. The vial was sealed, degassed by $\mathrm{N}_{2}$ and the mixture heated to $80^{\circ} \mathrm{C}$ for 3 hours. 


\section{Conditions 8: Neat in TEAF}

In a $5 \mathrm{~mL}$ vial, benzodiazepine 1 ( $37.3 \mathrm{mg}, 0.1 \mathrm{mmol}$ ) was dissolved in TEAF ( $2 \mathrm{~mL}$ ) followed by the addition of catalyst $(0.00625 \mathrm{mmol})$. The mixture was degassed by $\mathrm{N}_{2}$ and heated to $40^{\circ} \mathrm{C}$. The substrate did not dissolve entirely.

Results: (ee's in absolute values; TEAF 52/11 $=\mathrm{HCOOH}: \mathrm{Et}_{3} \mathrm{~N}$ in 5:2/1:1 mol:mol)

\begin{tabular}{|c|c|c|c|c|c|c|c|}
\hline \multirow{2}{*}{ Catalyst } & \multirow{2}{*}{ Conditions } & \multicolumn{2}{|c|}{ H-source } & \multirow{2}{*}{ solvent } & \multirow{2}{*}{$\begin{array}{c}t \\
\text { (h) }\end{array}$} & \multirow{2}{*}{ e.e. $(\%)$} & \multirow{2}{*}{ conv. (\%) } \\
\hline & & & (eq.) & & & & \\
\hline $\mathbf{F}$ & 1 & $\mathrm{HCOOH}$ & 5 & $\mathrm{MeOH}$ & 4.5 & 0 & 0 \\
\hline F & 1 & $\mathrm{HCOOH}$ & 10 & $\mathrm{MeOH}$ & 4.5 & 13 & 5 \\
\hline 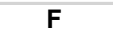 & 1 & $\mathrm{HCOOH}$ & 15 & $\mathrm{MeOH}$ & 4.5 & 14 & 36 \\
\hline C & 1 & $\mathrm{HCOOH}$ & 5 & $\mathrm{MeOH}$ & 4 & 0 & 0 \\
\hline C & 1 & $\mathrm{HCOOH}$ & 10 & $\mathrm{MeOH}$ & 4 & 42 & 8 \\
\hline C & 1 & $\mathrm{HCOOH}$ & 15 & $\mathrm{MeOH}$ & 4 & 39 & 23 \\
\hline $\mathbf{F}$ & 7 & IPA & solvent & IPA & 3 & 79 & 22 \\
\hline C & 2 & TEAF11 & 5.7 & $\mathrm{MeOH}$ & 4 & 59 & 15 \\
\hline C & 2 & TEAF52 & 5.7 & $\mathrm{MeOH}$ & 4 & 51 & 41 \\
\hline A & 2 & TEAF11 & 5.7 & $\mathrm{MeOH}$ & 3 & 54 & 9 \\
\hline C & 2 & TEAF11 & 5.7 & $\mathrm{MeOH}$ & 3 & 59 & 14 \\
\hline B & 2 & TEAF11 & 5.7 & $\mathrm{MeOH}$ & 3 & 37 & 3 \\
\hline I & 2 & TEAF11 & 5.7 & $\mathrm{MeOH}$ & 3 & 28 & 74 \\
\hline $\mathbf{J}$ & 2 & TEAF11 & 5.7 & $\mathrm{MeOH}$ & 3 & 35 & 19 \\
\hline $\mathbf{F}$ & 2 & TEAF11 & 5.7 & $\mathrm{MeOH}$ & 3 & 23 & 14 \\
\hline K & 2 & TEAF11 & 5.7 & $\mathrm{MeOH}$ & 3 & 7 & 3 \\
\hline G & 2 & TEAF11 & 5.7 & $\mathrm{MeOH}$ & 3 & 0 & 6 \\
\hline H & 2 & TEAF11 & 5.7 & $\mathrm{MeOH}$ & 3 & 71 & 12 \\
\hline $\mathbf{A}$ & 3 & TEAF11 & 5.7 & DCM & 17 & 11 & 25 \\
\hline C & 3 & TEAF11 & 5.7 & DCM & 17 & 18 & 29 \\
\hline B & 3 & TEAF11 & 5.7 & DCM & 17 & 7 & 14 \\
\hline$I$ & 3 & TEAF11 & 5.7 & DCM & 17 & 17 & 55 \\
\hline $\mathbf{J}$ & 3 & TEAF11 & 5.7 & DCM & 17 & 34 & 37 \\
\hline $\mathbf{F}$ & 3 & TEAF11 & 5.7 & DCM & 17 & 17 & 15 \\
\hline K & 3 & TEAF11 & 5.7 & DCM & 17 & 2 & 8 \\
\hline G & 3 & TEAF11 & 5.7 & DCM & 17 & 14 & 15 \\
\hline H & 3 & TEAF11 & 5.7 & DCM & 17 & 1 & 16 \\
\hline $\bar{A}$ & 8 & TEAF11 & solvent & TEAF11 & 17 & 15 & 1 \\
\hline A & 8 & TEAF52 & solvent & TEAF52 & 17 & 7 & 8 \\
\hline A & 6 & TEAF11 & 5.7 & $\mathrm{H} 2 \mathrm{O} / \mathrm{DCM}$ & 3 & 19 & 5 \\
\hline C & 6 & TEAF11 & 5.7 & $\mathrm{H} 2 \mathrm{O} / \mathrm{DCM}$ & 3 & 43 & 10 \\
\hline B & 6 & TEAF11 & 5.7 & $\mathrm{H} 2 \mathrm{O} / \mathrm{DCM}$ & 3 & 15 & 2 \\
\hline I & 6 & TEAF11 & 5.7 & $\mathrm{H} 2 \mathrm{O} / \mathrm{DCM}$ & 3 & 30 & 5 \\
\hline $\mathbf{J}$ & 6 & TEAF11 & 5.7 & $\mathrm{H} 2 \mathrm{O} / \mathrm{DCM}$ & 3 & 5 & 5 \\
\hline $\mathbf{F}$ & 6 & TEAF11 & 5.7 & H2O/DCM & 3 & 48 & 2 \\
\hline K & 6 & TEAF11 & 5.7 & $\mathrm{H} 2 \mathrm{O} / \mathrm{DCM}$ & 3 & 4 & 1 \\
\hline G & 6 & TEAF11 & 5.7 & H2O/DCM & 3 & 1 & 3 \\
\hline H & 6 & TEAF11 & 5.7 & $\mathrm{H} 2 \mathrm{O} / \mathrm{DCM}$ & 3 & 0 & 3 \\
\hline A & 5 & HCOONa & 10 & $\mathrm{H} 2 \mathrm{O} / \mathrm{DCM}$ & 3 & 47 & 6 \\
\hline H & 1 & $\mathrm{HCOOH}$ & 10 & $\mathrm{MeOH}$ & 3 & 57 & 19 \\
\hline H & 4 & HCOONa & 10 & $\mathrm{H} 2 \mathrm{O} / \mathrm{MeOH}$ & 3 & 45 & 2 \\
\hline H & 5 & HCOONa & 10 & $\mathrm{H} 2 \mathrm{O} / \mathrm{DCM}$ & 3 & 36 & 7 \\
\hline $\mathrm{H}$ & 2 & TEAF52 & 5.7 & $\mathrm{MeOH}$ & 3 & 63 & 29 \\
\hline
\end{tabular}




\section{First screening for the enantioselective hydrogenation of 1}

Procedure: Inside a $\mathrm{N}_{2}$ glovebox, the catalysts were prepared from $[\operatorname{Ir}(\mathrm{COD}) \mathrm{Cl}]_{2}(3.34 \mathrm{mg}, 0.005 \mathrm{mmol})$ in presence of the ligand $(1.05 \mathrm{eq} / \mathrm{Ir})$ for $1 / 2 \mathrm{~h}$ at $50^{\circ} \mathrm{C}$ in $\mathrm{DCM}(1 \mathrm{~mL}) .0 .05 \mathrm{~mL}$ of the catalyst solution was transferred via a liquid-handling robot into a $5 \mathrm{~mL}$ vial. For ligand L12, $1 \mathrm{eq} / \mathrm{Ir}$ of NaBArF was added to generate the cationic complex. lodine was added via the liquid handling robot from a $0.04 \mathrm{M}$ stock solution in DCM (2 eq/Ir). After evaporation of DCM, the substrate 1 was added as a solid $(18.6 \mathrm{mg}, 0.05 \mathrm{mmol})$ followed by the solvent (DCM, THF, TFE, $A c O H ; 1 \mathrm{~mL}$ ). The vials were capped and placed in the parallel hydrogenation reactor. The reactor was purged with $\mathrm{N}_{2}$ and pressurized with $\mathrm{H}_{2}$ (50 bar). The reaction was run overnight at room temperature. The samples for HPLC analysis were prepared by dissolving $100 \mu \mathrm{L}$ of the reaction mixture into $1 \mathrm{~mL}$ of $\mathrm{MeOH}: \mathrm{EtOH}(1: 1 \mathrm{v} / \mathrm{v})$.

\section{Ligand set:}

L1: (R)-(+)-2-[2-(Diphenylphosphino)phenyl]-4-isopropyl-2-oxazoline, CAS Number 164858-78-0

L2: (S)-(+)-2-[2-(Diphenylphosphino)phenyl]-4-phenyl-2-oxazoline, CAS Number 148461-15-8

L3: (S)-4-Isopropyl-2-[(S)-2-(bis(2-methoxyphenyl)phosphino)ferrocen-1-yl]oxazoline, Solvias- SL-N012-2

L4: (R)-diMe-PipPhos

L5: (S)-1-[(R)-2-(Dicyclohexylphosphino)ferrocenyl]ethyldiphenylphosphine, SL-J004-2, CAS Number 162291-01-2

L6:(R)-1-Dicyclohexylphosphino-2-[(R)-(N,N-dimethylamino)[2-(dicyclohexylphosphino)phenyl]methyl] ferrocene, SL-T002-1, CAS Number 1156547-61-3

L7: (R)-Phanephos, (R)-(-)-4,12-Bis(diphenylphosphino)-[2.2]-paracyclophane, CAS Number 364732-88-7

L8: (2S,3S)-(-)-Bis(diphenylphosphino)butane, ChiraPhos, CAS Number 64896-28-2

L9: (R)-2,2'-Bis[bis(3,5-di-tert-butyl-4-methoxyphenyl)phosphino]-6,6'-dimethoxy-1,1'-biphenyl, CAS Number 352655-61-9

L10: (2R,3R)-(-)-2,3-Bis(diphenylphosphino)-bicyclo[2.2.1]hept-5-ene, min. 95\% (R,R)-NORPHOS, CAS Number 71042-55-2

L11: (1S,1S',2R,2R')-1,1'-Di-tert-butyl-(2,2')-diphospholane, $\left(S, S^{\prime}, R, R^{\prime}\right)$-TangPhos, CAS Number 470480-321

L12: was obtained from the group of Pr. A. Pfaltz [See for example: Pauli, L.; Tannert, R.; Scheil, R.; Pfaltz, A. Asymmetric Hydrogenation of Furans and Benzofurans with Iridium-Pyridine-Phosphinite Catalysts. Chem. Eur. J., 2015, 21: 1482-1487.

Hydrogenation conditions: Ir $(0.0005 \mathrm{mmol}), \mathrm{I}_{2}$ (none or $\left.0.001 \mathrm{mmol}\right)$, substrate $1(0.05 \mathrm{mmol})$, Solvent (1mL), 50 bar $\mathrm{H}_{2}$, R.T., $18 \mathrm{~h}$. 
Results: See Scheme 2 in article and below ee $(>0=(S)$-major enantiomer).

\begin{tabular}{|c|c|c|c|c|c|c|c|c|c|c|c|c|c|}
\hline \multicolumn{2}{|l|}{ E.e. } & \multicolumn{12}{|c|}{ Catalysts } \\
\hline Additive & Solvent & L1 & L2 & L3 & L4 & L5 & L6 & L7 & L8 & L9 & L10 & L11 & L12 \\
\hline \multirow{4}{*}{ no 12} & DCM & -36 & -7 & -47 & 38 & -34 & 0 & 41 & 13 & 3 & -22 & -23 & 63 \\
\hline & THF & -27 & -10 & -13 & 18 & -57 & -4 & 18 & 1 & 27 & 10 & -9 & -28 \\
\hline & TFE & -21 & 0 & -75 & 55 & 22 & -18 & 46 & 0 & -42 & -4 & -52 & -35 \\
\hline & HOAc & 0 & 0 & 0 & -9 & 0 & 47 & 4 & 9 & -19 & 0 & 0 & 0 \\
\hline \multirow{4}{*}{12} & DCM & -56 & -6 & -61 & -38 & 2 & -3 & 6 & 18 & 6 & -13 & -39 & 26 \\
\hline & THF & -22 & -15 & -22 & -22 & -2 & -3 & 16 & 15 & -8 & -6 & -15 & -22 \\
\hline & TFE & 0 & 0 & 0 & 0 & 0 & 12 & 41 & 39 & -31 & -28 & 0 & -36 \\
\hline & HOAC & -40 & -27 & 0 & -7 & 27 & -3 & -1 & -6 & -25 & -2 & -33 & 0 \\
\hline
\end{tabular}




\section{4. $2^{\mathrm{d}}$ screening: 48 chiral ligands}

46 chiral ligands combined with $[\operatorname{Ir}(C O D) C l]_{2}$ and 2 preformed $\operatorname{Ir}(C O D) L B A r F$ with UBAPhox $(L 47, L 48)$ were tested in DCM as a solvent and with/without iodine as an additive.

\section{List of ligands:}

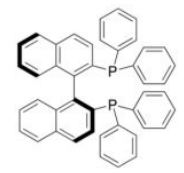

L13-(S)-Binap

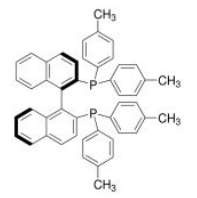

L14-(R)-Tol-Binap

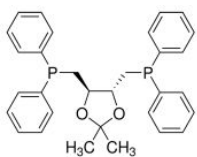

L15-(R, R)-DIOP

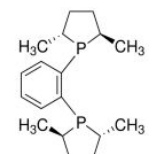

L16-(S, S)-MeDuPhos

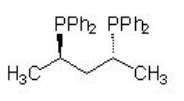

L17-(R, R)-BDPP

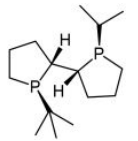

L18-(S,S,R,R)-TangPhos

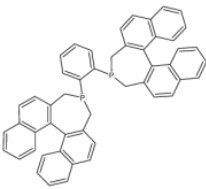

L19-(S)-BinaPhane

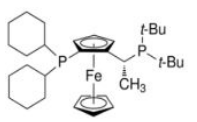

L20-JosiPhos (J009-1)

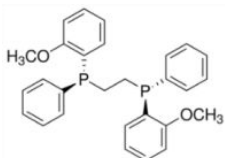

L21-(S, S)-DIPAMP

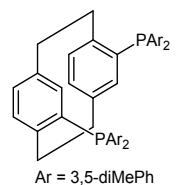

L22-Xylyl-PhanePhos

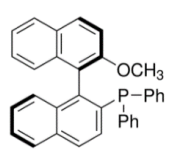

L23-( R)-MOP

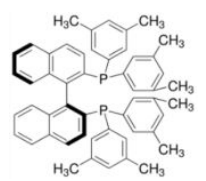

L24-(S)-Xylyl-Binap

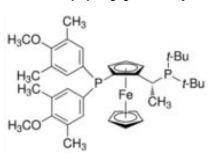

L25-JosiPhos 13-1

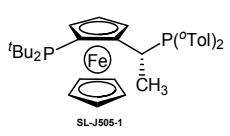

L26-JosiPhos 505-1

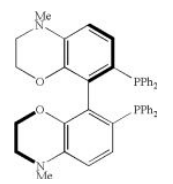

L27-(R)-SolPhos

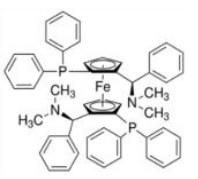

L28-MandyPhos M001

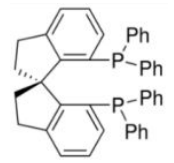

L29-(S)-SDP<smiles>CC(C(P(c1ccccc1)c1ccccc1)P(c1ccccc1)c1ccccc1)P(c1ccccc1)c1ccccc1</smiles>

L30-(2S,3S)-ChiraPhos

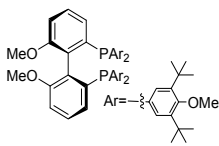

L31-(S)-BiPheP

$$
\text { (n) }
$$

L32-WalPhos (W003-2)

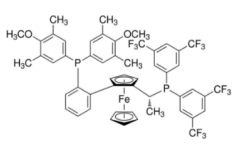

L33-WalPhos (W005-1)

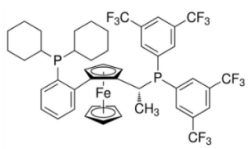

L34-WalPhos (W008-1)

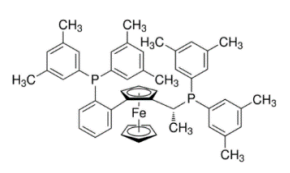

L35-WalPhos (W009-1)

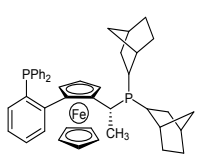

L36-WalPhos (W022-1)

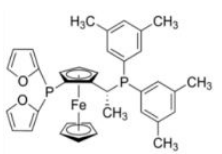

L37-JosiPhos (J015-1)

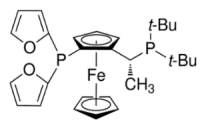

L38-JosiPhos (J212-1)

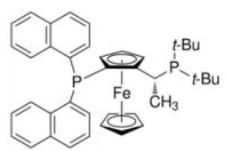

L39-JosiPhos (216-1)

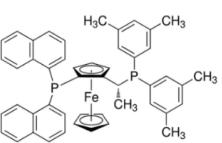

L40-JosiPhos (J404-1)

$\mathrm{H}_{3} \mathrm{CO}^{\mathrm{CH}_{3}} \mathrm{H}_{3} \mathrm{C} \mathrm{CH}^{\mathrm{CH}_{3}}$

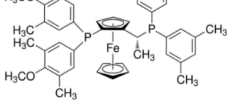

L41-JosiPhos (J418-1)

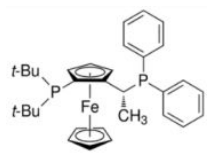

L42-JosiPhos (J502-1)

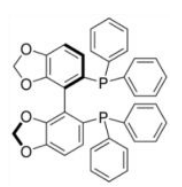

L43-(R )-SegPhos

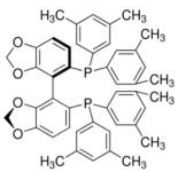

L44-( R)-DM-SegPhos

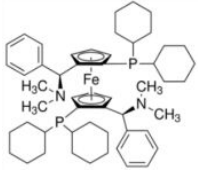

L45-MandyPhos (M002-2)

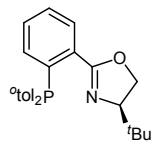

L46-Tol-tBu-Phox<smiles>CC(C)OC(Cc1ccccc1)(Cc1ccccc1)C1N=C(c2ccccc2)O[C@H]1C</smiles>

L47-UBAPhox<smiles>CC1OC(c2ccccc2)=NC1C(Cc1ccccc1)(Cc1ccccc1)Oc1ccccc1</smiles>

L48-UBAPhox

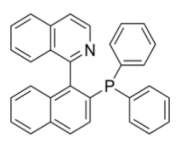

L49-( R)-Quinap

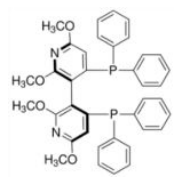

L50-(S)-P-Phos

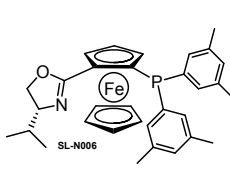

L51-POX-N006-2

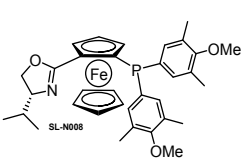

L52-POX-N008-2

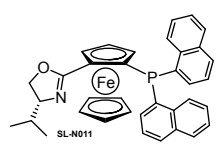

L53-POX-N011-2
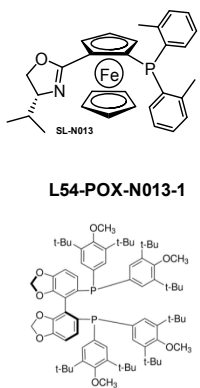

L55-( S)-DTBM-SegPhos

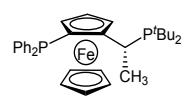

L56-JosiPhos 2-1

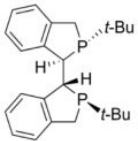

L57-(R,R,S,S)-DuanPhos

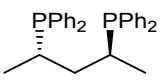

L58-(2S,4S)-SkewPhos

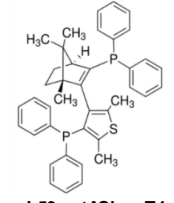

L59-catASium T1

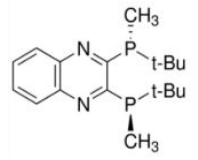

L60- (R, R)-QuinoxP* 
Procedure: The catalysts were formed as described above. With $\mathbf{L 4 7}$ and L48, the preformed (COD)IrLBArF complexes were used.

Library layout:

\begin{tabular}{|c|c|c|c|c|c|c|c|c|c|c|c|}
\hline \multicolumn{5}{|c}{ No lodine } & \multicolumn{5}{|c|}{ lodine } \\
\hline L13 & L21 & L29 & L37 & L45 & L53 & L13 & L21 & L29 & L37 & L45 & L53 \\
\hline L14 & L22 & L30 & L38 & L46 & L54 & L14 & L22 & L30 & L38 & L46 & L54 \\
\hline L15 & L23 & L31 & L39 & L47 & L55 & L15 & L23 & L31 & L39 & L47 & L55 \\
\hline L16 & L24 & L32 & L40 & L48 & L56 & L16 & L24 & L32 & L40 & L48 & L56 \\
\hline L17 & L25 & L33 & L41 & L49 & L57 & L17 & L25 & L33 & L41 & L49 & L57 \\
\hline L18 & L26 & L34 & L42 & L50 & L58 & L18 & L26 & L34 & L42 & L50 & L58 \\
\hline L19 & L27 & L35 & L43 & L51 & L59 & L19 & L27 & L35 & L43 & L51 & L59 \\
\hline L20 & L28 & L36 & L44 & L52 & L60 & L20 & L28 & L36 & L44 & L52 & L60 \\
\hline
\end{tabular}

Hydrogenation conditions: Ir $(0.0005 \mathrm{mmol}), \mathrm{I}_{2}$ (none or $\left.0.001 \mathrm{mmol}\right)$, substrate $1(0.05 \mathrm{mmol})$, Solvent (1mL), 50 bar $\mathrm{H}_{2}$, R.T., $18 \mathrm{~h}$.

Results: Conversion (\%) and enantiomeric excess (>0 = (S)-major enantiomer).

\begin{tabular}{|c|c|c|c|c|c|c|c|c|c|c|c|c|}
\hline Conversion & \multicolumn{6}{|c|}{ No lodine } & \multicolumn{6}{|c|}{ lodine } \\
\hline & A & B & C & D & $E$ & $\mathrm{~F}$ & G & $\mathrm{H}$ & 1 & $\mathrm{~J}$ & $\mathrm{~K}$ & L \\
\hline 1 & 1 & 3 & 52 & 30 & 10 & 0 & 100 & 81 & 99 & 100 & 22 & 33 \\
\hline 2 & 2 & 49 & 4 & 59 & 1 & 2 & 100 & 13 & 97 & 44 & 35 & 17 \\
\hline 3 & 92 & 1 & 19 & 19 & 33 & 49 & 100 & 22 & 99 & 12 & 51 & 100 \\
\hline 4 & 34 & 12 & 36 & 9 & 10 & 53 & 57 & 100 & 11 & 90 & 94 & 22 \\
\hline 5 & 11 & 38 & 32 & 40 & 0 & 42 & 99 & 19 & 84 & 100 & 16 & 99 \\
\hline 6 & 26 & 36 & 51 & 60 & 4 & 21 & 75 & 19 & 69 & 33 & 99 & 98 \\
\hline 7 & 38 & 11 & 74 & 1 & 3 & 1 & 100 & 100 & 79 & 100 & 100 & 98 \\
\hline 8 & 27 & 5 & 46 & 1 & 4 & 77 & 16 & 95 & 15 & 99 & 100 & 100 \\
\hline
\end{tabular}

\begin{tabular}{|c|c|c|c|c|c|c|c|c|c|c|c|c|}
\hline \multirow{2}{*}{ E.e. } & \multicolumn{9}{|c|}{ No lodine } & \multicolumn{6}{|c|}{ lodine } \\
\cline { 2 - 14 } & $\mathrm{A}$ & $\mathrm{B}$ & $\mathrm{C}$ & $\mathrm{D}$ & $\mathrm{E}$ & $\mathrm{F}$ & $\mathrm{G}$ & $\mathrm{H}$ & $\mathrm{I}$ & $\mathrm{J}$ & $\mathrm{K}$ & $\mathrm{L}$ \\
\hline 1 & -42 & -3 & 26 & -22 & -3 & -10 & 12 & 2 & -11 & -5 & 1 & -39 \\
\hline 2 & 14 & 39 & 25 & -15 & -41 & -7 & -7 & 30 & 20 & -24 & -52 & 30 \\
\hline 3 & 7 & 16 & -10 & 38 & 71 & 10 & 6 & 1 & -2 & 10 & 78 & 23 \\
\hline 4 & 31 & 13 & -83 & -33 & -55 & -41 & 39 & 10 & -13 & -18 & -85 & -19 \\
\hline 5 & -26 & -32 & -16 & -41 & -16 & 7 & 8 & -28 & 19 & -35 & -17 & -21 \\
\hline 6 & -23 & -61 & 32 & -54 & 8 & 23 & -39 & -24 & 11 & -37 & 41 & -8 \\
\hline 7 & -10 & -12 & -11 & 17 & 11 & 21 & 3 & -44 & 5 & -47 & -57 & 23 \\
\hline 8 & 9 & -51 & 73 & 38 & 8 & -38 & -5 & -38 & 1 & -50 & -63 & -32 \\
\hline
\end{tabular}




\section{5. $3^{\text {rd }}$ Screening at higher temperature}

14 chiral ligands combined with $[\operatorname{Ir}(C O D) C l]_{2}$ and 2 preformed $\operatorname{Ir}(C O D) L B A r F$ with UBAPhox $(L 47, L 48)$ were tested in THF, IPA, and EtOAC at $60^{\circ} \mathrm{C}$.

List of ligands:
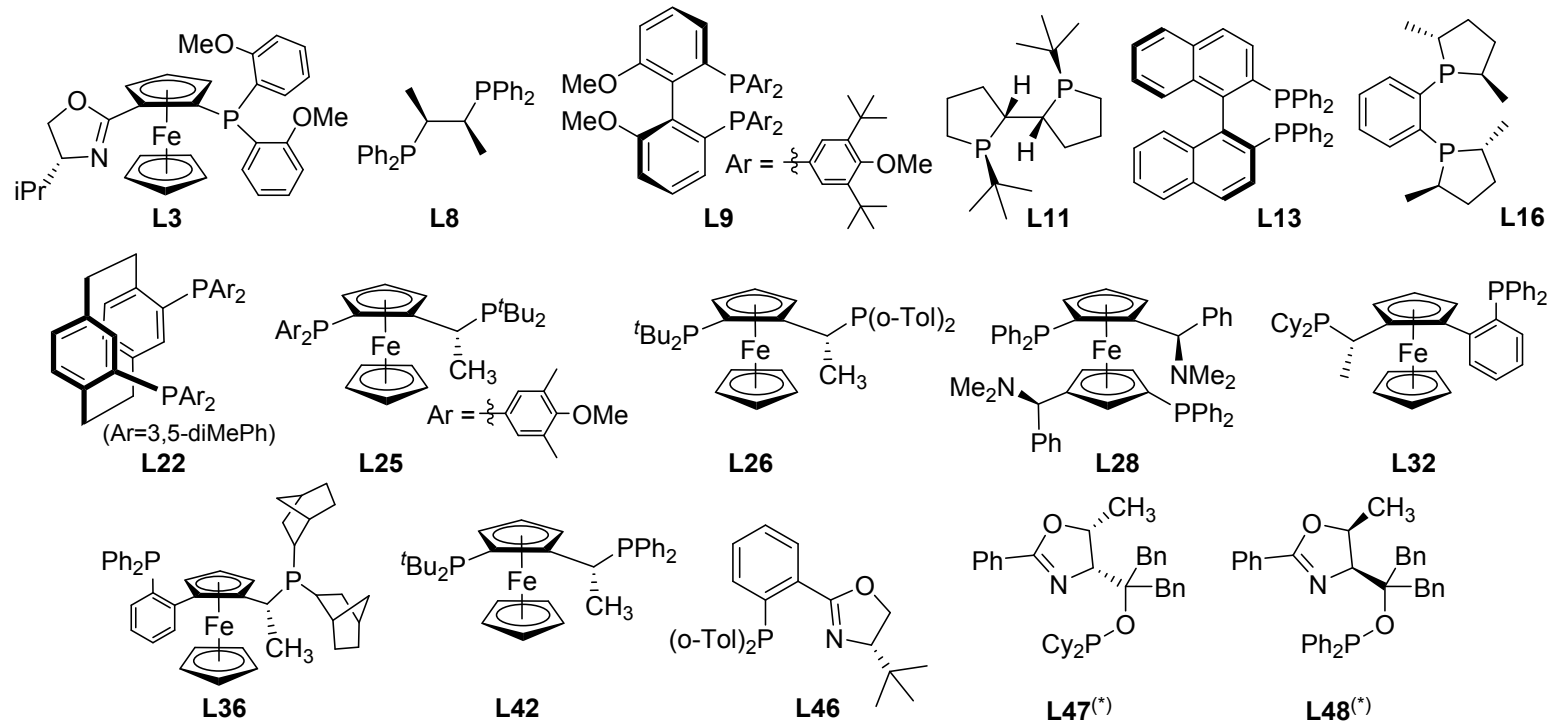

Procedure: The catalysts were formed as described above. With $\mathbf{L 4 7}$ and $\mathbf{L 4 8}$, the preformed (COD) IrLBArF complexes were used. Hydrogenation conditions: Ir $(0.0005 \mathrm{mmol}), \mathrm{I}_{2}$ (none or $\left.0.001 \mathrm{mmol}\right)$, substrate 1 (0.05mmol), Solvent ( $1 \mathrm{~mL}), 50 \mathrm{bar}_{2}, 60^{\circ} \mathrm{C}, 18 \mathrm{~h}$.

Results: (Positive ee $=(\mathrm{S})$-product is the major enantiomer)

\begin{tabular}{|c|c|c|c|c|c|c|c|c|c|c|c|c|}
\hline \multirow[b]{3}{*}{ Ligand } & \multicolumn{4}{|c|}{ THF } & \multicolumn{4}{|c|}{ IPA } & \multicolumn{4}{|c|}{ EtOAc } \\
\hline & \multicolumn{2}{|c|}{$\mathrm{No} \mathrm{I}_{2}$} & \multicolumn{2}{|c|}{$I_{2}$} & \multicolumn{2}{|c|}{$\mathrm{No} \mathrm{I}_{2}$} & \multicolumn{2}{|c|}{$I_{2}$} & \multicolumn{2}{|c|}{$\mathrm{No} \mathrm{I}_{2}$} & \multicolumn{2}{|c|}{$I_{2}$} \\
\hline & Conv. & e.e. & Conv. & e.e. & Conv. & e.e. & Conv. & e.e. & Conv. & e.e. & Conv. & e.e. \\
\hline L3 & 20 & -13 & 87 & -18 & 100 & -10 & 76 & -22 & 20 & 1 & 87 & -20 \\
\hline L8 & 50 & 2 & 100 & 5 & 100 & 3 & 100 & 1 & 42 & 8 & 100 & 9 \\
\hline L9 & 100 & 68 & 100 & 8 & 100 & 72 & 100 & -11 & 100 & 62 & 100 & -30 \\
\hline L11 & 100 & 25 & 100 & -14 & 100 & 36 & 100 & -7 & 100 & 29 & 100 & -16 \\
\hline L13 & 100 & -47 & 100 & 5 & 100 & -33 & 100 & 1 & 100 & -36 & 100 & 4 \\
\hline L16 & 100 & 38 & 100 & 7 & 100 & 3 & 100 & 3 & 100 & 3 & 100 & 17 \\
\hline L22 & 100 & 38 & 100 & 59 & 91 & 23 & 100 & -1 & 94 & 35 & 100 & 55 \\
\hline L25 & 100 & -7 & 100 & -16 & 87 & -9 & 72 & 5 & 100 & -4 & 100 & -30 \\
\hline L26 & 91 & -3 & 100 & -11 & 74 & -1 & 100 & -25 & 97 & -3 & 100 & -7 \\
\hline L28 & 49 & 6 & 100 & -32 & 59 & 5 & 100 & -7 & 50 & -10 & 100 & -23 \\
\hline L32 & 100 & 86 & 100 & 18 & 57 & 3 & 95 & 8 & 100 & 84 & 100 & 27 \\
\hline L36 & 92 & 77 & 100 & 4 & 61 & 0 & 92 & 3 & 100 & 77 & 100 & 11 \\
\hline L42 & 100 & -19 & 100 & -57 & 54 & 3 & 74 & -8 & 100 & -39 & 100 & -68 \\
\hline L46 & 57 & -2 & 100 & 0 & 86 & 10 & 82 & 0 & 32 & -14 & 100 & -7 \\
\hline L47 & 100 & 30 & 100 & 38 & 100 & 32 & 100 & 66 & 100 & 32 & 100 & 31 \\
\hline L48 & 100 & -38 & 100 & -61 & 100 & -28 & 100 & -60 & 100 & -28 & 100 & -67 \\
\hline
\end{tabular}




\section{Optimization of Ir/WalPhos}

During this study, both hands of WalPhos 003 were used indifferently as the focus was the catalyst activity and robustness. As a consequence, absolute values of ee's are given in the table below. WalPhos 003-1 is needed to produced the $(S)$-enantiomer of $\mathbf{2}$.

Substrate to catalyst ratio

Hydrogenation conditions: Ir (x mmol), substrate $1(0.05 \mathrm{mmol})$, Solvent $(1 \mathrm{~mL}), 50$ bar $\mathrm{H}_{2}, 60^{\circ} \mathrm{C}, 18 \mathrm{~h}$.

\begin{tabular}{|c|c|c|c|c|}
\hline$\#$ & S/C & Solvent & $\begin{array}{c}\text { e.e. } \\
\mathbf{( \% )}\end{array}$ & $\begin{array}{c}\text { conv. } \\
\mathbf{( \% )}\end{array}$ \\
\hline 1 & 100 & THF & 92 & 99 \\
\hline 2 & 200 & THF & 90 & 71 \\
\hline 3 & 500 & THF & 80 & 14 \\
\hline 4 & 1000 & THF & 65 & 3 \\
\hline 5 & 100 & toluene & 91 & 100 \\
\hline 6 & 200 & toluene & 91 & 83 \\
\hline 7 & 500 & toluene & 90 & 43 \\
\hline 8 & 1000 & toluene & 81 & 9 \\
\hline
\end{tabular}




\section{Acid screening}

Hydrogenation conditions: $\operatorname{Ir}(0.0005 \mathrm{mmol})$, substrate $1(0.05 \mathrm{mmol})$, Solvent $(1 \mathrm{~mL}), 50$ bar $\mathrm{H}_{2}, \mathrm{RT}, 18 \mathrm{~h}$.

\begin{tabular}{|c|c|c|c|c|}
\hline$\#$ & Acid (eq/1) & Solvent & $\begin{array}{c}\text { e.e. } \\
\text { (\%) }\end{array}$ & $\begin{array}{c}\text { conv. } \\
\text { (\%) }\end{array}$ \\
\hline 1 & $\mathrm{AcOH}$ ( 1 eq.) & THF & 92 & 54 \\
\hline 2 & Piv acid (1 eq.) & THF & 90 & 14 \\
\hline 3 & $\mathrm{HCOOH}$ (1 eq.) & THF & 90 & 39 \\
\hline 4 & $p$-Tos-OH (1 eq.) & THF & 22 & 95 \\
\hline 5 & $\mathrm{~B}\left(\mathrm{O}^{i} \mathrm{Pr}\right)_{3}(1$ eq.) & toluene & 96 & 52 \\
\hline 6 & $\mathrm{~B}(\mathrm{Et})_{3}(1$ eq.) & toluene & 95 & 70 \\
\hline 7 & $\mathrm{Ti}\left(\mathrm{O}^{i} \mathrm{Pr}\right)_{4}(1$ eq. $)$ & toluene & $\mathrm{N} / \mathrm{a}$ & 0 \\
\hline 8 & $\mathrm{Al}(\mathrm{TFE})_{3}(1$ eq.) & toluene & 39 & 65 \\
\hline 9 & $\mathrm{MeSO}_{3} \mathrm{H}(1$ eq.) & THF & 81 & 91 \\
\hline
\end{tabular}

\section{NMR study of the catalyst formation}

The formation of the precatalyst was studied by ${ }^{31} \mathrm{P}$ NMR. The following spectra in undeuterated DCM were acquired:

Spectrum A: Walphos in DCM ( $\left.{ }^{31} \mathrm{P}\left\{{ }^{1} \mathrm{H}\right\}(300 \mathrm{Mhz}, \mathrm{DCM}, 298 \mathrm{~K}) 12.08(\mathrm{PCy}),-15.13\left(\mathrm{PPh}_{2}\right)\right)$

Spectrum B: $[\operatorname{Ir}(\mathrm{COD}) \mathrm{Cl}]_{2}+$ Walphos @ RT for $10 \min \left({ }^{31} \mathrm{P}\left\{{ }^{1} \mathrm{H}\right\}(300 \mathrm{Mhz}, \mathrm{DCM}, 298 \mathrm{~K}) 29.12(\mathrm{PCY}),-15.01\right.$ $\left.\left(\mathrm{PPh}_{2}\right)\right)$

Spectrum C: $[\operatorname{Ir}(C O D) C l]_{2}+$ Walphos @ 50 ${ }^{\circ} \mathrm{C}$ for 0.5h $\left({ }^{31} \mathrm{P}\left\{{ }^{1} \mathrm{H}\right\}\right.$ (300 Mhz, DCM, 298K) $29.12\left(\mathrm{PC} \mathrm{y}_{2}\right),-15.01$ $\left.\left(\mathrm{PPh}_{2}\right)\right)$

Spectrum D: $[\operatorname{Ir}(\mathrm{COD}) \mathrm{Cl}]_{2}+$ Walphos @ 50 $\mathrm{C}$ for 15h $\left({ }^{31} \mathrm{P}\left\{{ }^{1} \mathrm{H}\right\}(300 \mathrm{Mhz}, \mathrm{DCM}, 298 \mathrm{~K}) 29.12(\mathrm{PCY}),-15.01\right.$ $\left.\left(\mathrm{PPh}_{2}\right),-13.194,55.708\right)$

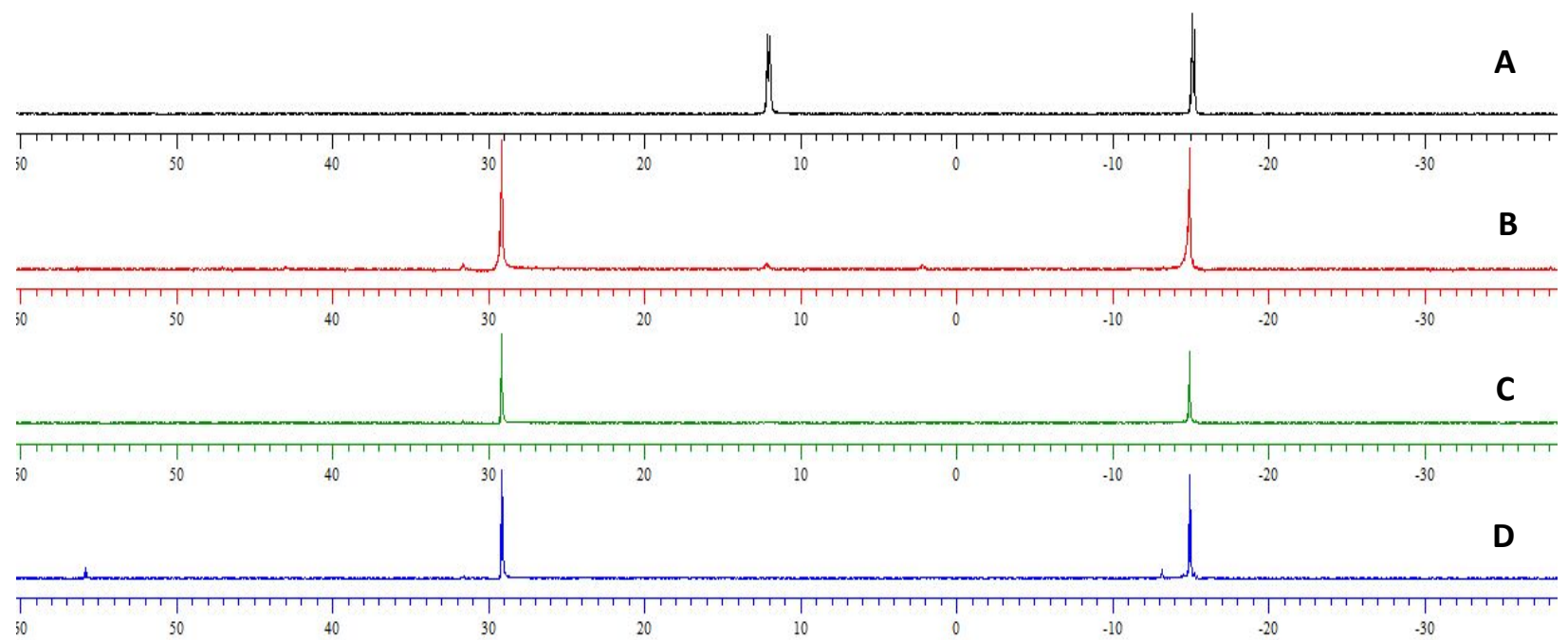


Remarkably, we can observe that the formation of the chelate does not happen even after one night at $50^{\circ} \mathrm{C}$. After $10 \mathrm{~min}$ at room temperature, a new Ir complex is formed where only the $-\mathrm{PCy}_{2}$ moiety of the ligand is bound to the $\mathrm{Ir}$ (shift of the signal from $12.08 \mathrm{ppm}$ to $29.12 \mathrm{ppm}$ while the $-\mathrm{PPh}_{2}$ signal remained unchanged) such as shown on the proposed structure below.

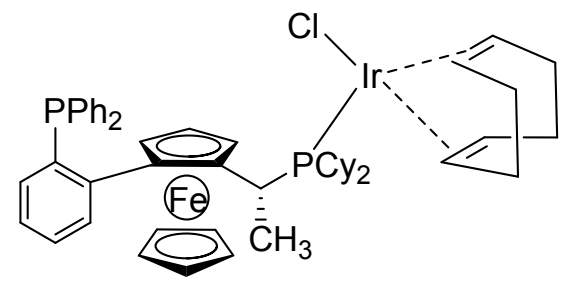

The complete chelation of the ligand may occur under $\mathrm{H}_{2}$ after removal of the COD. An additional spectrum taken after addition of substrate ( 5 eq) and Boc anhydride ( 5 eq) did not show any change either.

\section{Production campaign}

After transfer to Bayer, a set of scale-up experiments confirmed that this catalytic asymmetric hydrogenation reaction could be a cost-efficient method to cleanly convert $\mathbf{1}$ to target compound $\mathbf{S - 2}$ in good enantioselectivity (enantiomeric ratio 96:4).

$\mathrm{H}_{2} \mathrm{O}$ was detrimental to the reaction in $\mathrm{THF}$, but did not lead to significant catalyst deactivation in toluene. Using Boc anhydride as additive, catalyst performance was increased ( $\mathrm{S} / \mathrm{C}$ ratio). Toluene is also a preferred solvent for a robust hydrogenation process at high dilution.

However, THF increases solubility of reaction components. Upon scale-up, THF therefore seemed better suited for running more concentrated hydrogenation reactions. In addition, the lower boiling point of THF allowed for larger option space for solvent exchange. Although $\mathrm{S} / \mathrm{C}$ ratio was not optimal, we believe that the catalyst is sufficiently robust to be used for multi-kg scale production.

Sufficient stability of the reaction components allowed processing in the hydrogenation plant without prior preparation and addition of the catalyst solution under inert atmosphere. All solids were combined (starting material, Ligand and Ir-precatalyst), suspended in THF and transferred to the autoclave without special precaution to exclude air contact. Inertisation took place in the autoclave before introduction of hydrogen. The catalyst was only activated by hydrogenation of COD after complete inertisation.

For the ease of processing, telescoping conversion to the desired product by hydrogenation in THF and subsequent crystallization from isopropanol was chosen for scale-up. 


\section{Optimized procedure}

Hydrogenation $(2 \mathrm{x})$

Benzodiazepine 1 (1.200 kg), Walphos SL-W003-1 (33.2 g, 1.5 mol\%, Solvias) and [Ir(COD)Cl] 2 (16.3 g, 0.75 mol\%, Umicore) were placed in a stirable vessel at ambient temperature. THF ( $9 \mathrm{~L}$ ) was added and the vessel was inertized with nitrogen. The resulting thin suspension was transferred to a $20 \mathrm{~L}$ pressure reactor (Büchi, Hastelloy ${ }^{\circledR}$ ), additional THF ( $3 \mathrm{~L}$ ) was added to complete transfer of solids from transfer lines. The reactor was pressurized with hydrogen ( $80 \mathrm{bar}$ ), and the mixture was heated to $50^{\circ} \mathrm{C}$. After 16 hours, the reactor was cooled to $23^{\circ} \mathrm{C}$, the atmosphere was exchanged to nitrogen and the crude hydrogenation solution (2: $93.8 \%$ area, enantiomeric ratio 96.1: 3.9 (S:R) by HPLC) was transferred to a storage vessel.

Crystallization

The crude hydrogenation solutions from two pressure reactions were combined for crystallization in a 36 L stainless steel reactor.

After filtration, the solvent was distilled $\left(40^{\circ} \mathrm{C} / 200 \mathrm{mbar}\right)$ to ca. $7.2 \mathrm{~kg}$ solution (ca. 3 -fold solution in THF). The solution was cooled to $23-27^{\circ} \mathrm{C}$, and $i-\mathrm{PrOH}$ ( $14.4 \mathrm{~L}$, ca. 6 -fold) was slowly added (ca. $175 \mathrm{~mL} / \mathrm{min}$ ) over $90 \mathrm{~min}$. The resulting suspension was stirred for $16 \mathrm{~h}$, filtered, washed with $i$-PrOH ( 3 times $1.2 \mathrm{~L}$ ) and dried $\left(50^{\circ} \mathrm{C}\right.$, vacuum) to yield $1.677 \mathrm{~kg}$ of $(S)-2$ in (70\% yield, enantiomeric ratio 99.5:0.5 by HPLC, $99 \%$ ee) as off-white crystalline solid.

\section{Analytical data}

(4S)-1-(4-bromophenyl)-7,8-dimethoxy-4-methyl-4,5-dihydro-3H-2,3-benzodiazepine (S-2):

${ }^{1} \mathrm{H}$ NMR (400 MHz, DMSO-d $)_{6} \delta$ ppm $1.13(\mathrm{~d}, J=6.4 \mathrm{~Hz}, 3 \mathrm{H}) 2.62(\mathrm{dd}, J=13.7,6.5 \mathrm{~Hz}, 1 \mathrm{H}) 2.86(\mathrm{dd}, J=13.8$, $3.0 \mathrm{~Hz}, 1 \mathrm{H}) 3.55(\mathrm{~s}, 3 \mathrm{H}) 3.80(\mathrm{~s}, 3 \mathrm{H}) 3.82-3.98(\mathrm{~m}, 1 \mathrm{H}) 6.51(\mathrm{~s}, 1 \mathrm{H}) 6.89(\mathrm{~s}, 1 \mathrm{H}) 7.05$ (d, J=3.3 Hz, $1 \mathrm{H})$ $7.37(\mathrm{~m}, \mathrm{~J}=8.4 \mathrm{~Hz}, 2 \mathrm{H}) 7.51(\mathrm{~m}, \mathrm{~J}=8.4 \mathrm{~Hz}, 2 \mathrm{H})$.

LCMS: $\mathrm{Rt}=9.62 \mathrm{~min} ; \mathrm{MS}$ (ESIpos): $\mathrm{m} / \mathrm{z}=375.0666(\mathrm{M}+\mathrm{H})^{+}$

MS: ThermoFisherScientific LTQ-Orbitrap-XL; HPLC: Agilent 1200SL; column: Agilent, POROSHELL 120, $3 \mathrm{x}$ $150 \mathrm{~mm}, \mathrm{SB}-\mathrm{C} 182.7 \mu \mathrm{m}$; Eluent A: 1 I water + 0.1\% trifluroacetic acid; Eluent B: 1 I acetonitrile $+0.1 \%$ trifluroacetic acid; Gradient: $0.0 \mathrm{~min} 2 \% \mathrm{~B} \rightarrow 1.5 \mathrm{~min} 2 \% \mathrm{~B} \rightarrow 15.5 \mathrm{~min} 95 \% \mathrm{~B} \rightarrow 18.0 \mathrm{~min} 95 \% \mathrm{~B}$; Oven: $40^{\circ} \mathrm{C}$; Flow: $0.75 \mathrm{ml} / \mathrm{min}$; UV-detection: $210 \mathrm{~nm}$

\section{REFERENCES}

(1) This reactor was developed by Premex in cooperation with DSM. See: www.premexreactorag.ch/e/spezialloesungen/produkteneuheiten/ 
(2) Preparation of 1: Siegel, S.; Baeurle, S.; Cleve, A.; Haendler, B.; Fernandez-Montalvan, A. E.; Moenning, U.; Krause, S.; Lejeune, P.; Busemann, M.; Kuhnke, J. Bicyclo 2,3-benzodiazepines and spirocyclically substituted 2,3benzodiazepines PCT Int. Appl. WO 2014/128067 A1, 28 August 2014, Example 45A. 